\title{
Automatic Image Enhancement by Artificial Bee Colony Algorithm
}

\author{
Adiljan Yimit ${ }^{1}$, Yoshihiro Hagihara ${ }^{2}$, Tasuku Miyoshi ${ }^{2}$, Yukari Hagihara ${ }^{3}$ \\ ${ }^{1}$ Graduate School of Engineering, Iwate University, Morioka, Japan \\ ${ }^{2}$ Department of Mechanical Engineering, Iwate University, Morioka, Japan \\ ${ }^{3}$ Department of Electrical Engineering and Computer Science, Iwate University, Morioka, Japan
}

\begin{abstract}
With regard to the improvement of image quality, image enhancement is an important process to assist human with better perception. This paper presents an automatic image enhancement method based on Artificial Bee Colony (ABC) algorithm. In this method, $\mathrm{ABC}$ algorithm is applied to find the optimum parameters of a transformation function, which is used in the enhancement by utilizing the local and global information of the image. In order to solve the optimization problem by $\mathrm{ABC}$ algorithm, an objective criterion in terms of the entropy and edge information is introduced to measure the image quality to make the enhancement as an automatic process. Several images are utilized in experiments to make a comparison with other enhancement methods, which are genetic algorithm-based and particle swarm optimization algorithm-based image enhancement methods.
\end{abstract}

Keywords: Image enhancement, artificial intelligent optimization, Artificial Bee Colony algorithm.

\section{INTRODUCTION}

Image enhancement is an important process with respect to the improvement of image quality in image processing and analysis system, such as medical image analysis, computer vision applications and remote sensing, etc. The objective of this process is to make improvements in the appearance and visual quality of image in order to assist human with better perception and interpretability of information or provide more useful input for other automated image processing systems [1]. Up to now, different types of image enhancement methods have been proposed, and in general, most of these are spatial domain-based and/or frequency domain-based methods.

In recent years, nature inspired optimization algorithms have received particular interest in image enhancement due to the simplicity and flexibility [2-6]. In [2], Poli and Cagnoni proposed a global contrast enhancement technique. In their approach, genetic programming was utilized to adapt the color map in the image so as to fit the demands of the human interpreter. Later, Munteanu and Lazarescu [3] presented an image enhancement technique using a real coded GA with a subjective evaluation criterion to globally adapt the gray-level intensity transformation in the image. And in [4], Saitoh proposed a similar enhancement method. However, in his method, the fitness was given by an objective criterion in respect to the number of edges in the image and a clumping factor of the intensity transformation curve. Then, Munteanu and Rosa [5] used the combination of different transformation functions with different parameters to produce the enhanced image by GA. Recently particle swarm optimization (PSO) algorithm was also used in the image enhancement [6].

In this paper, an automatic image enhancement method was presented by using the same criterion function as proposed by Munteanu and Rose [7]. The proposed method made use of a swarm intelligence algorithm, named Artificial Bee Colony algorithm [8], which is similar to existing nature inspired algorithms, to optimize the parameters of transformation function. The reason for choosing this algorithm is that it has been proved to be better than GA and PSO algorithms with respect to the numerical function optimization problems [9] and is seldom studied in image enhancement. The rest of this paper is organized as follows: in section 2, ABC algorithm is briefly reviewed. Section 3 presents the proposed method. Section 4 reports on the experimental results from some images and discussions. We draw some conclusions in Section 5. 


\section{ARTIFICIAL BEE COLONY ALGORITHM}

Recently, similar to the existing nature inspired algorithms, a new mimic algorithm inspired by the behaviors of bees, named Artificial Bee Colony algorithm, was proposed by Karaboga and Basturk [8]. In [9], it has been shown that ABC algorithm is superior to other optimization algorithms such as GA, PSO and Differential Evolution (DE), etc. Due to the simplicity and robustness of $\mathrm{ABC}$ algorithm, it has been implemented to solve various problems in image processing, robot path planning, parameter identification, job-shop scheduling.

In $\mathrm{ABC}$ algorithm, the colony of artificial bees is composed of three types of bees: employed bees, onlooker bees and scouts. A bee searching for a food source is called an employed bee, and a bee waiting on the dance area to choose a food source it should go to is an onlooker bee. A scout is a bee that carries out a random search. Half of the colony involves the onlookers, and the other half consists of the employed bees. The number of employed bees equals that of the food sources, and one employed bee chooses only one food source around the hive. When the foraging process of the bee colony starts, the locations of a set of food sources are randomly chosen by the bees from a given area around the hive, and the nectar amounts of these sources are estimated. After the bees come back to the hive with the nectar information, they share the information of the food sources with the onlooker bees, which are waiting on the dance area. Then, the bee colony enters a cycle of searching iteration. Each cycle of searching iteration involves following three steps: sending the employed bees onto the food sources and then measuring their nectar amounts; selecting of the food sources by the onlookers after sharing the information of employed bees and determining the nectar amount of the foods; determining the scout bees and then sending them onto possible food sources.

Considering that an optimization problem is regarded as a foraging process of bee colony, the position of a food source denotes a possible solution of this problem, and the nectar amount of a food source represents the quality (fitness) of the associated solution. Then, the main steps of $\mathrm{ABC}$ algorithm with respect to the optimization problem can be described as follows:

(1) Initialize every solution $x_{i}$ of the bee colony and measure the fitness $f_{i}$ of $x_{i}$.

(2) Produce a new solution $y_{i}$ by employed bee in the neighbor of $x_{i}$ using the following equation:

$$
y_{i}=x_{i}+\phi\left(x_{i}-x_{k}\right),
$$

where $\phi$ is a random real number in $[-1,1], x_{k}$ is a neighbor of $x_{i}$.

(3) Replace the solution $x_{i}$ with $y_{i}$ if the fitness of $x_{i}$ is smaller than the fitness of $y_{i}$.

(4) Generate a candidate solution by the onlooker in the neighborhood based on the probability $p_{i}$ computed by the following equation:

$$
p_{i}=f_{i} / \sum_{i=1}^{S N} f_{i},
$$

where $\mathrm{SN}$ is a total number of the solutions and $f_{i}$ is the fitness of the solution $x_{i}$.

(5) Update the solutions by the greedy criteria.

(6) Memorize the best solution and the corresponding fitness.

(7) Replace the old solutions with the new generated solutions if the old solutions reached the abundant condition.

(8) Repeat steps (2)-(7), until the maximum number of iterations is reached or stop conditions are satisfied.

\section{PROPOSED METHOD}

In the proposed method, there are two important factors that influence the quality of the enhanced image. One is a transformation function, which will generate new pixel intensities for enhanced image from original image. The other is an objective evaluation criterion, which is indispensable to automatically measure the quality of the produced image.

\subsection{Transformation Function}

Image enhancement can be carried out by using many different types of mechanisms. In these mechanisms, grayscale transformation is considered as one of the fundamental processes. It can be applied by utilizing the information of the image locally or globally. In contrast to the global enhancement, the local one applies transformation functions that are based on the gray-level distribution in the neighborhood of each pixel in the given image. There is a traditional enhancement technique presented in [10], which transforms the intensity value of each pixel $f(x, y)$ to $g(x, y)$ by using the 
information of local area centered at $(x, y)$ with the combination of global information described by the following function:

$$
g(x, y)=\left[\frac{M}{\sigma(x, y)}\right] \cdot[f(x, y)-m(x, y)],
$$

where $M$ is an average intensity of the entire image, and $\sigma(x, y)$ and $m(x, y)$ are the mean and standard deviation in a neighborhood centered at $(x, y)$, respectively.

However, directly applying the above function on the certain image gives the same result until the size of the neighborhood changes. Therefore, Manteano and Rose [7] redefined (1) to make the transformation function more controllable for producing different enhanced results by adding some parameters and additional part. The redefined transformation function is given as follows:

$$
g(x, y)=\kappa\left[\frac{M}{\sigma(x, y)+b}\right] \cdot[f(x, y)-c \cdot m(x, y)]+m(x, y)^{a},
$$

where $a, b, c$, and $\kappa$ are the real positive parameters and they are the same for the whole image. From (4), it is obvious that (3) can be considered as the special case of (4) for $\mathrm{b}=0, \mathrm{c}=1, \kappa=1$, and the last term $m(x, y)^{a}$ equal to 0 . The redefined function can extend the distribution of the transformation output range for certain image, which is different from the original transformation function. By this way, the best qualified image can be found by getting an optimal combination of these four parameters.

\subsection{Objective Criterion}

The certain goal of this proposed method is to make an automatic enhancement, in which no human intervention at all is necessary for the entire process. To achieve this goal, there should be an objective criterion which automatically determines if the quality of image is good or not. In this paper, we use an objective criterion function presented in [7]. It is formed by combining three performance measures in terms of the entropy value, the sum of edge intensities and the number of pixels on the edges. According to the Ref. [10], good enhanced image has more number of edge pixels compared to the original image and enhanced version should have a higher intensity of the edges [4]. But these two are not sufficient to test an enhanced image and that is why one more measure has been taken i.e. entropy value of the image. Entropy value reveals the information content in the image. If the distribution of the intensities is uniform, then we can say that histogram is equalized and the entropy of the image will be more [11]. The objective criterion function can be described as

$$
F(x)=\log (\log (E(I(x)))) \cdot \frac{n_{-} \text {edgels }(I(x))}{M \times N} \cdot H(I(x)),
$$

where $F(\mathrm{x})$ is the criterion function, the higher value of which corresponds to the better enhanced image; $I(x)$ is the enhanced image obtained by transforming the original image with equation (4), and $M \times N$ is the size of the image. $H(I(x))$ is a measure of the entropy in the image $I(x) . E(I(x))$ is the sum of the intensity of the edges detected with a Sobel edge detector; $n$ edgels $(I(x))$ is the number of pixels on the edges, where the edges are the products of applying an automatic threshold detection [12] after applying the Sobel edge detector on the enhanced image.

\subsection{ABC-based Enhancement Methodology}

In the automatic enhancement method, the task of finding the best enhanced image is a searching process of the optimal parameters $(a, b, c$ and $\kappa)$ for the transformation function. Accordingly, when applying $\mathrm{ABC}$ algorithm to solve the searching problem, the tuning process of the parameters in the equation (4) can be considered as a foraging process of bee colony. The positions of food sources represent the possible sets of values of parameters and the nectar amount (fitness) of the food source correspond to the quality of the enhanced image, where the quality is computed from the objective criterion function defined in equation (5).

The procedure of the proposed method is briefly described as bellow. Initially, SN number of solutions, which are 4 dimensional vectors (with respect to the parameters of $a, b, c$ and $\kappa$ within a specific range), are randomly generated, and their fitness values are computed from the enhanced images produced by utilizing the parameters of each solution; Then, until the iteration finishes, the new solution is generated from each existed solution by the employed bee and its probability value corresponding to the fitness value is computed for the generation of a candidate solution, which is 
carried out by the onlooker. The fitness value of the candidate solution is also calculated to make an update on all solution. In each iteration, the best solution and its fitness value are memorized. Finally, when the entire process has finished, the enhanced image is obtained by the solution with the maximum fitness value.

\section{EXPERIMENTAL RESULTS AND DISCUSSION}

In order to demonstrate the performance of the proposed method, we have tested a set of various images. In the paper, only three of them, with gray levels $L=256$, were used to draw comparisons with other methods. These original images are "Girl", "Kid" and "City" with the size of $256 \times 256,240 \times 180$ and $400 \times 400$, labeled (a) in Figures 1 to 3 respectively. All the original images have different types of gray level histograms. Two other methods, which are the GA-based [7] and PSO-based [6] image enhancement methods, were employed to make a comparison. The reason for choosing the two methods is that they both are nature inspired optimization-based methods. In all the methods, the neighborhood size for computing the local mean and local standard deviation was chosen as $3 \times 3$. Objective evaluation was carried out by employing two measures, namely Detail variance (DV) and Background Variance (BV). Both are computed by calculating the variances of the local neighborhood of each pixel. If the local variance of the pixel is greater than a threshold, then the average variance of that of pixels is named DV; otherwise the average variance is named BV. According to Ref. [13], it can be said that a good enhanced image has the higher DV value and the lower BV value, while compared to the input image.

Table $1 \mathrm{DV}$ and BV values of the enhanced images.

\begin{tabular}{|c|c|c|c|c|c|c|c|c|}
\hline \multirow{2}{*}{ Images } & \multicolumn{2}{|c|}{ Original } & \multicolumn{2}{c|}{ GA-based } & \multicolumn{2}{c|}{ PSO-based } & \multicolumn{2}{c|}{ Proposed } \\
\cline { 2 - 9 } & $\mathrm{DV}$ & $\mathrm{BV}$ & $\mathrm{DV}$ & $\mathrm{BV}$ & $\mathrm{DV}$ & $\mathrm{BV}$ & $\mathrm{DV}$ & $\mathrm{BV}$ \\
\hline Girl & 117.071 & 0.677 & 232.555 & 0.561 & 167.549 & 0.763 & 201.196 & 0.791 \\
\hline Kid & 268.087 & 0.071 & 287.066 & 0.0 .099 & 282.224 & 0.691 & 373.356 & 0.051 \\
\hline City & 151.286 & 0.038 & 82.845 & 0.647 & 211.262 & 0.674 & 195.623 & 0.673 \\
\hline
\end{tabular}

The images labeled (b) to (d) in Figures 1 to 3 show the enhanced results from the original images, and their corresponding DV and BV values are tabulated in Table 1. From the table, it is clear that the proposed method gave the better results while compared to the other methods. Observing the enhanced images, it can be easy to find that all the results produced by the proposed method are enhanced efficiently. For the "Girl" image, all the methods gave similar results; the PSO-based method and the proposed method produced better results than GA-based method, even if their DV
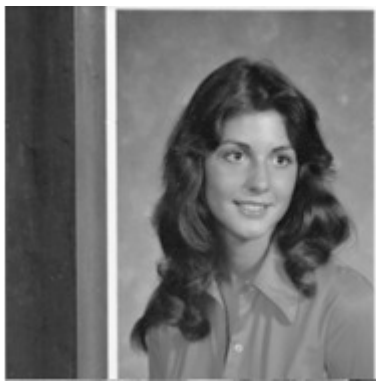

(a)
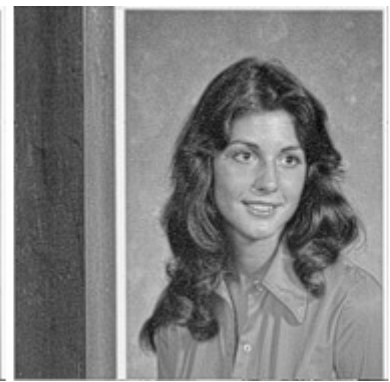

(b)
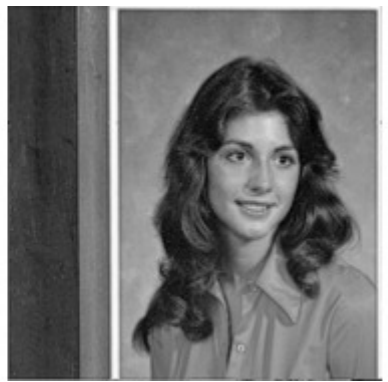

(c)
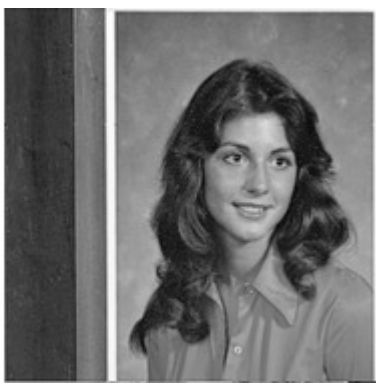

(d)

Figure 1. "Girl" image (a) Original image, (b) GA-based enhanement, (c) PSO-based enhancement, (d) proposed enhancment

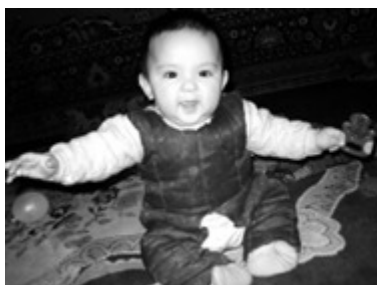

(a)

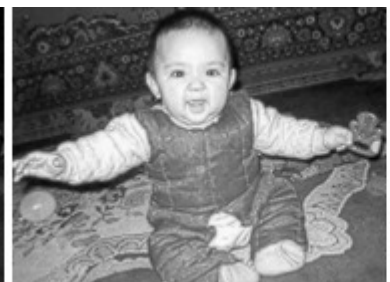

(b)

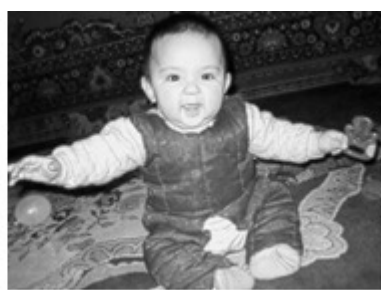

(c)

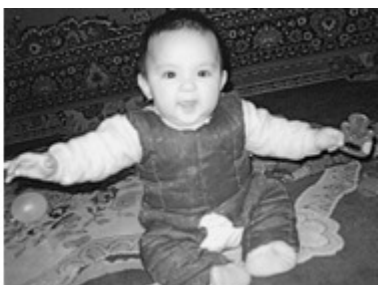

(d)

Figure 2. "Kid" image (a) Original image, (b) GA-based enhanement, (c) PSO-based enhancement, (d) proposed enhancment 


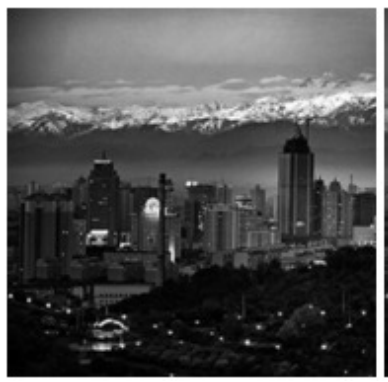

(a)

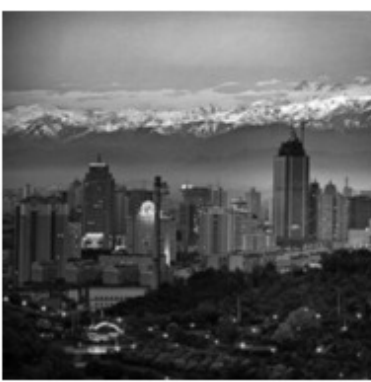

(b)

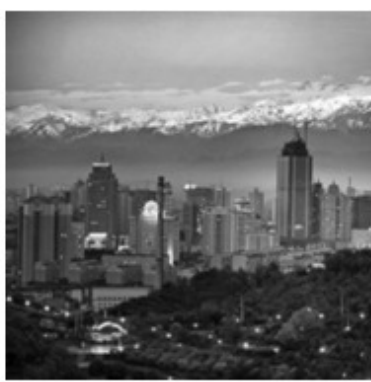

(c)

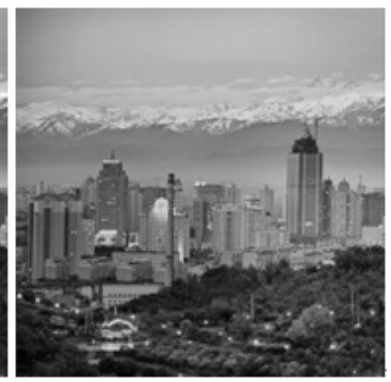

(d)

Figure 3. "City" image (a) Original image, (b) GA-based enhanement, (c) PSO-based enhancement, (d) proposed enhancment

values were lower than GA-based method. For "Kid" and "City" images, the proposed method yielded the best enhanced results, especially as regards the edges. According to the results of the above three images and other experimental images, we concluded that our proposed method can produce better enhancement.

\section{CONCLUSIONS}

In this paper, an automatic image enhancement method using Artificial Bee Colony was presented. This method regards finding the optimum set of parameters of transformation function as a search process with the help of an objective criterion, which is a fitness function proportional to the entropy of the entire image and to the intensity of edges in the image. Experimental results of the proposed method are compared with other two enhancement methods, which are genetic algorithm-based and particle swarm optimization-based methods. In most of the cases, the presented method gives the better results.

\section{REFERENCES}

[1] F. Y. Shih, Image Processing and Pattern Recognition: Fundamentals and Techniques, John Wiley \& Sons, N.J. (2010).

[2] R. Poli and S. Cagnoni, "Evolution of pseudo-coloring algorithms for image enhancement," Univ. Birmingham, Birmingham, Tech. Rep. CSRP-97-5 (1997).

[3] C. Munteanu and V. Lazarescu, "Evolutionary contrast stretching and detail enhancement of satellite images," Proc. Mendel, Berno, 94-99 (1999).

[4] F. Saitoh, "Image contrast enhancement using genetic algorithm," Proc. IEEE SMC, Tokyo, 899-904 (1999).

[5] C. Munteanu and A. Rosa, "Evolutionary image enhancement with user behavior modeling," ACM SIGAPP Applied Computing Review. 9 (1), 8-14 (2001).

[6] M. Braik, A. Sheta and A. Ayesh, "Image Enhancement Using Particle Swarm Optimization," Proc. WCE. 1, London (2007).

[7] C. Munteanu and A. Rosa, "Gray-scale enhancement as an automatic process driven by evolution," IEEE Trans. on Systems, Man and Cybernatics. 34 (2), 1292-1298 ( 2004).

[8] D. Karaboga, “An Idea Based on Bee Swarm for Numerical Optimization,” Tech. Rep.-TR06 (2005).

[9] D. Karaboga and B. Basturk, "On the performance of artificial bee colony (ABC) algorithm", Applied Soft Computing. 8 (1), 687-697 (2008).

[10] R. C. Gonzales and P. Winter, Digital Image Processing, 2nd Ed, Addison Wesley (1987).

[11] A. K. Jain, Fundamentals of Digital Image Processing, Prentice Hall, Englewood Cliffs, N.J. (1989).

[12] P. L. Rosin, "Edges: saliency measures and automatic thresholding," Machine Vision and Applications. 9, 139-159 (1997).

[13] G. Ramponi, N. Strobel, S. K. Mitra and T. H. Yu, "Nonlinear Unsharp Masking Methods for Image Contrast Enhancement," Journal of Electronic Imaging. 5 (3), 353-366 (1996). 